Gasco Txabarri, J. (2016). El empleo de estrategias en el aprendizaje de las matemáticas en enseñanza secundaria obligatoria. Revista de Investigación Educativa, 34(2), 487-502.

DOI: http://dx.doi.org/10.6018/rie.34.2.222901

\title{
El empleo de estrategias en el aprendizaje de las Matemáticas en Enseñanza Secundaria Obligatoria
}

\author{
The use of strategies for the learning of Mathematics in \\ Compulsory Secondary Education
}

Javier Gasco Txabarri

UPV-EHU. España

\begin{abstract}
Resumen
El empleo de estrategias en el aprendizaje de las Matemáticas tiene repercusión en el razonamiento y en la resolución de problemas, entre otros aspectos. El objetivo de este estudio es detectar diferencias que se puedan producir en el empleo de dichas estrategias en función del curso académico. Para ello se han recogido datos de 565 estudiantes de 2. $\stackrel{\circ}{ }$ 3. .0 y $4 .{ }^{\circ}$ curso de Educación Secundaria Obligatoria (ESO). La herramienta empleada para tal fin ha sido una adaptación del Motivated Strategies for Learning Questionnaire (MSLQ). Los resultados obtenidos indican un aumento del empleo de la mayoría de estrategias a medida que el curso es superior; la excepción tan solo ocurre en la estrategia de repetición. La investigación previa no es unánime a la hora de definir una tendencia evolutiva del uso de estrategias en el aprendizaje de las Matemáticas en Educación Secundaria. Los datos aportados en este artículo pretenden contribuir a la compresión de la diversidad estratégica del alumnado.

Palabras clave: educación matemática; estrategias de aprendizaje; diferencias de curso; enseñanza secundaria.
\end{abstract}

\begin{abstract}
The use of learning strategies in Maths education has an impact on reasoning and problem solving, among other aspects. The aim of this study is to detect differences in the use of these strategies according to the academic grade. These data were collected from 565 students of the

Correspondencia: Javier Gasco Txabarri, Escuela Universitaria de Magisterio de Vitoria-Gasteiz. C/ Juan Ibáñez de Santo Domingo, 1. 01006-Vitoria-Gasteiz (Alava) (España). E-mail: javier.gasco@ehu.es
\end{abstract}


2nd, 3rd and 4th grade of Compulsory Secondary Education. The tool used for this purpose was adapted from the Motivated Strategies for Learning Questionnaire (MSLQ). The results indicate an increase in the employment of the majority of strategies as the grade is superior; the only exception occurs with the strategy of rehearsal. Previous research is not unanimous in defining an evolutionary trend of the use of strategies for the learning of Mathematics in Secondary Education. The data provided in this article are intended to contribute to the understanding of the student's strategic diversity.

Keywords: Mathematical education; learning strategies; Differences among grades; Compulsory Secondary Education.

\section{Introducción}

Desde mediados del siglo XX las estrategias de aprendizaje se han considerado herramientas fundamentales en el proceso de enseñanza-aprendizaje en todas las áreas de conocimiento. Inicialmente, estas estrategias se asociaron con el procesamiento cognitivo de la información, lo que dio pie a la construcción de modelos que permitieran comprender el funcionamiento de la cognición humana (Badia, Álvarez, Carretero, Liesa y Becerril, 2012).

Emplear estrategias de aprendizaje conlleva la puesta en marcha de un aprendizaje estratégico y autónomo, basado en el concepto de "aprender a aprender" (Nisbet y Shucksmith, 1986) y puesto en valor por la Unión Europea (European Commission, 2006). Desde esta perspectiva, el metaaprendizaje es el nexo de unión entre las estrategias de aprendizaje y la autorregulación (Thoutenhoofd y Pirrie, 2013). De hecho, en ciertas investigaciones, los términos de aprendizaje autorregulado y de estrategias de aprendizaje se emplean indistintamente (Dinsmore, Alexander y Loughlin, 2008). Las similitudes son manifiestas si se define la autorregulación del aprendizaje como los procesos individuales mediante los cuales el alumnado activa, orienta y mantiene sus pensamientos, afectos y acciones hacia la consecución de objetivos de aprendizaje (Monereo y Badia, 2013).

La transcendencia educativa de las estrategias de aprendizaje lleva a relacionarlas con importantes variables psicoinstruccionales como son la motivación escolar (GarcíaGarcía, Biencinto-López, Carpintero-Molina, Núñez-del-Río y Arteaga- Martínez, 2013; Gil-Jaurena, 2012) y el autoconcepto (Núnez, González-Pienda, García, GonzálezPumariega y García, 1998).

Existen diversos enfoques y líneas de investigación sobre estrategias de aprendizaje; no obstante, en cuanto a sus implicaciones en el aprendizaje, la mayoría de los estudios coinciden en la importancia de fomentar entre el alumnado la formación y desarrollo de estrategias cognitivas, metacognitivas, de autorregulación personal, motivacional y de aprendizaje cooperativo con el fin de mejorar su rendimiento escolar (Zimmerman, 1994; Pintrich, 1995).

Asumir que las estrategias de aprendizaje puedan ser el motor del aprendizaje confiere especial importancia a su clasificación; quizás por esta razón no exista un consenso sobre su configuración dimensional, a lo que hay que añadir un carácter multifacético que hace de las estrategias un constructo difuso, en ocasiones. 


\section{Clasificaciones y medidas para las estrategias}

Como se ha mencionado anteriormente, no hay unanimidad a la hora de precisar las estrategias que se emplean para mejorar el aprendizaje. Sin embargo, esta confusión aparente se suaviza al comprobar que la mayoría de modelos explicativos pivotan sobre tres grandes conjuntos de estrategias: las estrategias cognitivas, las metacognitivas y las afectivas.

Gargallo (2012) propone una categorización en cuatro grupos de estrategias: (i) estrategias afectivas, disposicionales y de apoyo; (ii) estrategias metacognitivas, de regulación y control; (iii) estrategias de búsqueda, recogida y selección de información; y (iv) estrategias de procesamiento y uso de la información adquirida. Cada una de ellas presenta una serie de escalas más específicas.

Badia et al. (2012) consideran las estrategias como procesos cognitivos, como procesos de toma de decisiones situados y dependientes del sistema educativo y, por último, como competencias para la resolución satisfactoria de problemas de aprendizaje. En este último caso, el de las competencias, se tiene en cuenta la metacognición y la regulación de los aprendizajes, la dimensión afectiva y los contextos auténticos de aprendizaje (contextos cultural y social).

Otra herramienta ampliamente implementada en infinidad de estudios es el cuestionario MSLQ (Motivated Strategies for Learning Questionnaire) de Pintrich, Smith, García y Mckeachie (1991). Su estructura principal está formada por dos grandes bloques: las estrategias de aprendizaje y la motivación. En lo que respecta a las estrategias, se diferencian estrategias cognitivas, metacognitivas y contextuales.

\section{Las estrategias en el aprendizaje de las Matemáticas}

El principal objetivo de las investigaciones sobre estrategias de aprendizaje se ha focalizado en el análisis de la variación del rendimiento académico en función del empleo de las mismas; este hecho se produce en cualquier área o ciclo educativo, también en Matemáticas (Ahmed, van der Werf, Kuyper y Minnaert, 2013; Murayama, Pekrum, Lichtenfeld y vom Hofe, 2013).

En lo que respecta a las relaciones entre las estrategias y el rendimiento en Matemáticas, un estudio sobre los resultados de PISA 2003 concluye que la importancia de la estrategia de elaboración (por ejemplo, establecer conexiones con áreas relacionadas o buscar soluciones alternativas) se incrementa a medida que el nivel de rendimiento aumenta (Thiessen y Blasius, 2008). Además, se ponen en cuestión las dimensiones establecidas al comprobarse que, al menos en el área de las estrategias de aprendizaje de las Matemáticas, el número de dimensiones extraídas varía de forma sistemática (de una a tres) en cada país en función del nivel de rendimiento en Matemáticas.

Una investigación análoga con datos del informe PISA 2000 (Chiu, Wing-Yin y McBride-Chang, 2007) informa que: (1) El empleo de la estrategia de repetición (o memorización de conceptos) está asociado negativamente con el rendimiento en Matemáticas, coincidiendo este resultado con las conclusiones obtenidas por otro informe (Czuchry y Dansereau, 1998) en el sentido de que memorizar es una estrategia ineficaz para el aprendizaje de nociones nuevas; (2) La estrategia de elaboración no tiene relación con el 
rendimiento, no concordando este resultado con otros estudios realizados anteriormente que relacionan directamente ambas variables, probablemente porque el cuestionario no distingue adecuadamente entre las estrategias de repetición y de elaboración existiendo una alta correlación entre ambas; (3) El empleo de estrategias metacognitivas correlaciona positivamente con el rendimiento en Matemáticas.

Otra línea de investigación en lo concerniente al aprendizaje de las Matemáticas y las estrategias de aprendizaje es la intervención en el aula mediante la enseñanza del empleo de dichas estrategias, sobre todo poniendo en valor la influencia de la instrucción metacognitiva (Zamani y Mir, 2011). En lo que respecta a estrategias cognitivas, según un estudio realizado con alumnado del último curso de Educación Primaria (Perels, Dignath y Schmitz, 2009), el entrenamiento en el uso de estrategias de aprendizaje en la clase de Matemáticas da resultados satisfactorios. Estos resultados se complementan con otro estudio realizado con alumnado de 2.ํำ de la ESO; se certifica la mejora de las competencias a través de la formación fuera de la escuela combinando la autorregulación y la resolución de problemas matemáticos (Perels, Günter y Schmitz, 2005).

Escasean las investigaciones en relación a las diferencias entre los cursos de Educación Secundaria en el empleo de estrategias en el aprendizaje de las Matemáticas. Los pocos resultados publicados no siempre son coincidentes, a lo que hay que añadir el inconveniente de que no siempre estudian la evolución de los mismos cursos (Liu, 2009; Marugán, Carbonero, León y Galán, 2013; Zimmermann y Martínez-Pons, 1990).

\section{Metodología}

El objetivo del presente estudio se centra en detectar posibles diferencias en el empleo de estrategias de aprendizaje con el fin de poder comprender la diversidad existente entre cursos de la ESO.

\section{Muestra}

En esta investigación participan 631 estudiantes de tres cursos de Educación Secundaria Obligatoria (ESO): 2. . de la ESO (13-14 años, 32 \%), 3.ํ de la ESO (14-15 años, 37 \%) y 4. ํ de la ESO (15-16 años, $31 \%$ ), de los cuales 336 (53.3\%) eran mujeres y 295 (46.7 \%) hombres. Por motivos de errores u omisiones en las respuestas a los cuestionarios, la muestra final quedó reducida a 565 sujetos. La recogida de datos se ha realizado en 8 centros educativos que pertenecen a la Comunidad Autónoma Vasca, integrantes tanto de la red pública (5) como de la red privada concertada (3). Tras contactar con varios centros, finalmente se seleccionan aquellos que responden positivamente a la oferta de participación, por lo que se trata de una muestra de conveniencia.

La totalidad del alumnado que se encuentra en el aula en el momento de la realización cumplimenta el cuestionario. Además, en cada centro educativo, todos los grupos de los cursos seleccionados participan en la prueba. 
En la Tabla 1 se expone la distribución de la muestra en función del curso académico y del sexo:

Tabla 1

Distribución de la muestra por cursos y sexos

\begin{tabular}{|c|c|c|c|}
\hline \multirow{2}{*}{ CURSO } & \multicolumn{2}{|c|}{ SEXO } & \multirow[b]{2}{*}{ Total } \\
\hline & Mujer & Hombre & \\
\hline 2. ${ }^{\circ} \mathrm{ESO}$ & 95 & 81 & 176 \\
\hline 3. ${ }^{\circ} \mathrm{ESO}$ & 111 & 88 & 199 \\
\hline 4. ${ }^{\circ} \mathrm{ESO}$ & 100 & 90 & 190 \\
\hline Total & 306 & 259 & $N=565$ \\
\hline
\end{tabular}

\section{Instrumentos de medida}

Con el objetivo de medir las estrategias de aprendizaje del alumnado en este estudio exploratorio se han propuesto 25 ítems (ver anexo). El cuestionario se basa en una versión para Enseñanza Secundaria del MSLQ (Motivated Strategies for Learning Questionnaire) (Pintrich, Smith, Garcia y McKeachie, 1991) adaptado por Berger y Karabenick (2011) para ajustarse al estudio de las Matemáticas.

La consistencia interna de la versión original en lengua inglesa de este cuestionario se evaluó mediante la ro de Raykov obteniendo valores entre .58 y .86 (Berger y Karabenick, 2011) en una investigación realizada con 306 sujetos del curso equivalente a 3.. de la ESO (9th grade). Dicha estructura, de 33 ítems en total, estaba compuesta por tres escalas de medida para las estrategias cognitivas (repetición, organización y elaboración), una escala para medir las estrategias metacognitivas y dos escalas contextuales (el manejo del tiempo-espacio de estudio y los recursos de ayuda).

Sin embargo, un estudio posterior, que pretendía confirmar la estructura factorial del cuestionario, concluye que el ajuste escalar se optimiza considerando siete escalas (Wernke, Wagener, Anschuetz y Moschner, 2011). Coincidiendo con dicho análisis, el estudio factorial exploratorio del cuestionario castellano aquí propuesto, se ajusta mejor a dicha estructura heptafactorial formada por las mismas escalas del original pero dividiendo la escala de estrategias metacognitivas en dos: planificación y seguimientoregulación; esta nueva propuesta suprime ocho ítems formando un total de 25 . Los ítems se responden en base a una escala Likert de cinco puntos (desde "completamente en desacuerdo" hasta "completamente de acuerdo"). A continuación se concreta la configuración de la citada versión castellana (tres dimensiones divididas en siete escalas):

- Estrategias cognitivas

- Repetición (3 ítems): Se miden las estrategias para aprender por repetición. Por ejemplo: "Para estudiar Matemáticas repito varias veces las fórmulas o definiciones con el fin de memorizarlas". 
- Organización (2 ítems): Se tienen en cuenta las maneras de gestionar los aprendizajes matemáticos. Por ejemplo: "Estudio Matemáticas haciendo diagramas, cuadros o tablas para organizar lo que he aprendido".

- Elaboración (2 ítems): Se mide por una parte cómo se relaciona el aprendizaje en Matemáticas con otras materias y por otra la transformación de los conceptos matemáticos para una mejor comprensión personal. Por ejemplo: “Cuando estudio Matemáticas intento relacionar lo nuevo con lo que ya sé".

- Estrategias metacognitivas

Se distribuyen en dos escalas: la planificación y el seguimiento-regulación.

- Planificación (5 ítems): Se recopila información sobre cómo se planifican los estudios. Por ejemplo: "Antes de empezar a estudiar Matemáticas planeo qué y cómo voy a hacerlo".

- Seguimiento (4 ítems): Contiene ítems relativos a la conciencia, conocimiento y control que tiene el educando sobre su propia cognición. Por ejemplo: "Cuando estudio Matemáticas llevo un control de lo que he aprendido".

- Regulación (3 ítems): Se refiere a la habilidad para controlar el esfuerzo y la atención frente a las distracciones o ante tareas difíciles. Por ejemplo: "Si tengo dificultades para resolver un problema, intento otras formas para resolverlo".

- Estrategias contextuales y de gestión de recursos

- Recursos de ayuda (3 ítems): Mide a quién o a qué se recurre en caso de dificultad en el aprendizaje. Por ejemplo: "Si no entiendo algo en Matemáticas pido ayuda a otros estudiantes".

- Entorno de estudio-tiempo de estudio (3 ítems): Se refiere a las costumbres de estudio en cuanto a espacio y horario. Por ejemplo: "Estudio Matemáticas en un entorno en el que puedo concentrarme".

\section{Procedimiento}

Con el fin de realizar la recogida de datos, se solicita una cita con la dirección de cada centro elegido. Una vez obtenido el visto bueno, se expone tanto la naturaleza de la investigación como las pruebas propuestas al profesorado de Matemáticas de 2. ${ }^{\circ}$, 3. ${ }^{\circ}$ y $4 .^{\circ}$ de la ESO. Cuando el profesorado acepta realizar la prueba en su aula, se acuerda una hora lectiva para llevarla a cabo.

Se advierte de que la prueba es anónima y que los datos personales que se requieren son la fecha de nacimiento y el sexo, exclusivamente. Además, se informa de que los resultados obtenidos no influirán en la calificación escolar.

Dependiendo de cada individuo, la realización de la prueba dura entre 10 y 15 minutos.

\section{Análisis de datos}

Primeramente se realiza el análisis factorial exploratorio que permite comprobar la pertenencia de cada ítem a su escala correspondiente. Seguidamente se mide la consistencia interna de las escalas mediante el $\alpha$ de Cronbach.

Para analizar las diferencias entre cursos se implementa la prueba no paramétrica para k muestras independientes de Kruskal-Wallis. Para realizar los test post-hoc se 
ha empleado la prueba no paramétrica U de Mann-Withney. La razón de la elección de dichas pruebas no paramétricas es que los datos recogidos no cumplen los criterios ni de normalidad ni de homocedasticidad.

En las pruebas para el análisis de dos muestras independientes, concretamente en el test U de Mann-Withney, se ha calculado el tamaño del efecto (effect size), denotado por el parámetro $\mathrm{r}$. La interpretación del coeficiente es la siguiente: $\mathrm{r}=.10$, tamaño del efecto débil; $\mathrm{r}=.30$, tamaño del efecto moderado; $\mathrm{y}$ a partir de $\mathrm{r}=.50$ tamaño del efecto fuerte ( $\mathrm{r}$ toma valores entre 0 y 1) (Field, 2009).

Todos los datos obtenidos han sido guardados en una tabla de Excel. El análisis estadístico se ha efectuado empleando el software estadístico PASW Statistics 18.

\section{Resultados}

Los resultados obtenidos en la presente investigación se clasifican en tres apartados: el análisis factorial exploratorio del cuestionario y el análisis de las diferencias en función de los cursos analizados $\left(2^{\circ}, 3^{\circ}\right.$ y $4^{\circ}$ de la ESO).

\section{Análisis factorial exploratorio}

Se procede a realizar el análisis factorial exploratorio. Las correlaciones entre las escalas son positivas aunque algunas no lo suficientemente altas $(r<.40)$, por lo que se decide efectuar el análisis de componentes principales con rotación equamax. El índice $\mathrm{KMO}=.96$ es suficiente y la prueba de esfericidad de Bartlett muestra que la relación entre escalas es significativa. Los siete factores explican el $71.74 \%$ de la varianza. Los datos se presentan en la Tabla 2:

Tabla 2

Análisis factorial exploratorio

\begin{tabular}{|c|c|c|c|c|c|c|c|}
\hline \multirow{2}{*}{ Ítems } & \multicolumn{7}{|c|}{ Factores/Estrategias } \\
\hline & Se-Re & $\mathrm{Pl}$ & En & $\mathrm{El}$ & Ay & $\mathrm{O}$ & $\mathrm{R}$ \\
\hline R1 & & & & & & & .85 \\
\hline $\mathrm{R} 2$ & & & & & & .53 & .65 \\
\hline R3 & & & & & & & .81 \\
\hline O1 & & .34 & .40 & & & .55 & \\
\hline $\mathrm{O} 2$ & & .38 & & & & .66 & \\
\hline El1 & & & & .87 & & & \\
\hline El2 & & & & .73 & & & \\
\hline Pl1 & & .73 & & & & & \\
\hline $\mathrm{Pl} 2$ & & .61 & & .47 & & & \\
\hline $\mathrm{Pl} 3$ & .37 & .53 & & & & & \\
\hline Pl4 & & .60 & .31 & .36 & & & \\
\hline $\mathrm{Pl} 5$ & & .45 & .30 & .33 & & & \\
\hline
\end{tabular}




\begin{tabular}{|c|c|c|c|c|c|c|c|}
\hline Se1 & .54 & .30 & & .39 & & & \\
\hline Se2 & .49 & .41 & & .36 & & & \\
\hline Se3 & .47 & .30 & .40 & & & & \\
\hline Se 4 & .58 & & .30 & & .34 & .33 & \\
\hline Re1 & .47 & & .33 & .46 & & & \\
\hline Re2 & .65 & & & & .45 & & \\
\hline Re3 & .67 & & & .31 & & & \\
\hline Ay1 & & & & & .84 & & \\
\hline Ay2 & & & & & .72 & & \\
\hline Ay3 & .46 & & .31 & & .51 & & \\
\hline En1 & & & .46 & .34 & & .41 & \\
\hline En2 & & .32 & .67 & & & & \\
\hline En3 & & & .66 & & & .31 & \\
\hline $\begin{array}{l}\text { arianza } \\
\text { plicada }\end{array}$ & $11.85 \%$ & $11.24 \%$ & $10.49 \%$ & $11.44 \%$ & $9.44 \%$ & $9.36 \%$ & $7.92 \%$ \\
\hline
\end{tabular}

Nota: R: Repetición; O: Organización; El: Elaboración; Pl: Planificación; Se: Seguimiento; Re: Regulación; Se-Re: Seguimiento-Regulación; En: Entorno-tiempo de estudio; Ay: Recursos de ayuda.

Los resultados del análisis factorial exploratorio revelan una estructura de siete escalas o factores: tres estrategias cognitivas (repetición, organización y elaboración), dos metacognitivas (planificación y seguimiento-regulación) y dos contextuales y de gestión de recursos (Entorno-tiempo de estudio y recursos de ayuda).

La consistencia interna de las escalas, medida empleando el $\alpha$ de Cronbach, resulta aceptable; todas ellas presentan fiabilidades suficientes, iguales o superiores a .70 (repetición ( $\alpha=.71)$, organización ( $\alpha=.73)$, elaboración $(\alpha=.80)$, planificación $(\alpha=.86)$, seguimientoregulación $(\alpha=.92)$, Entorno-tiempo de estudio $(\alpha=.70)$ y recursos de ayuda $(\alpha=.70))$.

\section{Diferencias en función del curso académico}

Las diferencias en función del curso académico se han analizado efectuando la prueba no paramétrica de Kruskall-Wallis comparando las medias aritméticas logradas en cada estrategia (medias factoriales, por tanto) y en cada curso. Se muestran los datos en la Tabla 3:

Tabla 3

Diferencias en función del curso académico

\begin{tabular}{ccccccc}
\hline & & & \multicolumn{5}{c}{ Prueba de Kruskal-Wallis } \\
Estrategia & Curso & Media & Desv. típ. & $\chi^{2}$ & gl & p \\
\hline \multirow{3}{*}{ Repetición } & $2 .{ }^{\circ}$ ESO & 2.95 & .95 & & & \\
& $3 .{ }^{\circ}$ ESO & 2.89 & .99 & 3.12 & 2 & .202 \\
& $4 .^{\circ}$ ESO & 3.09 & .89 & & & \\
& $2 .{ }^{\circ}$ ESO & 2.11 & 1.10 & &
\end{tabular}




\begin{tabular}{|c|c|c|c|c|c|c|}
\hline \multirow[t]{3}{*}{ Organización } & 3..$^{\circ} \mathrm{ESO}$ & 2.71 & 1.12 & 46.20 & 2 & .000 \\
\hline & 4. ${ }^{\circ} \mathrm{ESO}$ & 2.84 & 1.09 & & & \\
\hline & 2. ${ }^{\circ} \mathrm{ESO}$ & 2.16 & 1.15 & & & \\
\hline \multirow[t]{3}{*}{ Elaboración } & 3..$^{\mathrm{e}} \mathrm{ESO}$ & 2.61 & 1.04 & 64.12 & 2 & .000 \\
\hline & 4..$^{\circ} \mathrm{ESO}$ & 3.11 & 1.11 & & & \\
\hline & 2. ${ }^{\mathrm{o}} \mathrm{ESO}$ & 2.21 & 1.00 & & & \\
\hline \multirow[t]{3}{*}{ Planificación } & 3..$^{\circ} \mathrm{ESO}$ & 2.48 & .84 & 27.99 & 2 & .000 \\
\hline & 4. ${ }^{\circ} \mathrm{ESO}$ & 2.74 & .99 & & & \\
\hline & 2. ${ }^{\circ} \mathrm{ESO}$ & 2.78 & 1.22 & & & \\
\hline \multirow[t]{3}{*}{$\begin{array}{l}\text { Seguimiento- } \\
\text { Regulación }\end{array}$} & 3. ${ }^{\circ} \mathrm{ESO}$ & 3.38 & .88 & 25.69 & 2 & .000 \\
\hline & 4. ${ }^{\circ} \mathrm{ESO}$ & 3.39 & .99 & & & \\
\hline & 2. ${ }^{\mathrm{o}} \mathrm{ESO}$ & 3.05 & .85 & & & \\
\hline \multirow[t]{3}{*}{ Recursos ayuda } & 3..$^{\circ} \mathrm{ESO}$ & 3.76 & .94 & 63.97 & 2 & .000 \\
\hline & 4. ${ }^{\circ} \mathrm{ESO}$ & 3.47 & .78 & & & \\
\hline & 2. ${ }^{\circ} \mathrm{ESO}$ & 2.62 & 1.03 & & & \\
\hline \multirow[t]{2}{*}{ Entorno estudio } & 3. ${ }^{\circ} \mathrm{ESO}$ & 3.10 & .97 & 26.60 & 2 & .000 \\
\hline & 4. ${ }^{\mathrm{o}} \mathrm{ESO}$ & 3.10 & .96 & & & \\
\hline
\end{tabular}

Las diferencias son estadísticamente significativas en todas las estrategias exceptuando en la de repetición ( $\mathrm{p}>.050)$, estrategia cognitiva consistente en memorizar lo aprendido.

A continuación se han realizado los test post-hoc $U$ de Mann-Withney con el fin de estudiar las diferencias dos a dos entre cursos. Los resultados entre los cursos 2. o $^{\circ}$ y 3.ํ se exponen en la Tabla 4:

Tabla 4

Diferencias post-hoc entre los cursos $2 .^{\circ}$ y $3 .^{\circ}$

\begin{tabular}{ccccc}
\hline & \multicolumn{4}{c}{ Test U de Mann-Withney } \\
\hline Estrategia & $\mathrm{U}$ & $\mathrm{Z}$ & $\mathrm{p}$ & $\mathrm{r}$ \\
Organización & 1192550 & 5.44 & .000 & .28 \\
Elaboración & 13657.50 & 3.78 & .000 & .19 \\
Planificación & 14125.00 & 3.30 & .010 & .17 \\
Seguimiento-Regulación & 12991.50 & 4.31 & .000 & .22 \\
Recursos ayuda & 9626.00 & 7.62 & .000 & .39 \\
Entorno estudio & 12805.50 & 4.57 & .000 & .24 \\
\hline
\end{tabular}

Las diferencias estadísticamente significativas se producen en todas las estrategias evaluadas; además, en todas ellas, el alumnado de 3. obtiene puntuaciones superiores en comparación con el alumnado de 2.ำ (ver tabla 3). 
La estrategia de organización tiene en cuenta las maneras de gestionar los aprendizajes matemáticos. La estrategia cognitiva de elaboración mide cómo se relaciona el saber matemático con otras materias, además de la transformación que se realiza de los conceptos matemáticos para una mejor comprensión de los mismos. La planificación y el seguimiento-regulación, ambas estrategias metacognitivas, miden la preparación, el control y el conocimiento que tiene el alumnado sobre su propia cognición. La estrategia de recursos de ayuda se refiere a la búsqueda de ayuda en caso de dificultad y el entorno de estudio evalúa el ambiente elegido para estudiar.

En todos los tipos de estrategias el tamaño del efecto es entre débil y moderado por el hecho de que el parámetro $\mathrm{r}$ se mueve entre .10 y .30, a excepción de los recursos de ayuda en el que es alto ( $r>30)$.

En la Tabla 5 se exponen las diferencias en el empleo de estrategias entre los cursos de $3 . \stackrel{\circ}{y}$ de $4 . \stackrel{\circ}{*}$

Tabla 5

Diferencias post-hoc entre los cursos 3. ${ }^{\circ}$ y $4 .^{\circ}$

\begin{tabular}{ccccc}
\hline & \multicolumn{4}{c}{ Test U de Mann-Withney } \\
\hline Estrategia & $\mathrm{U}$ & $\mathrm{Z}$ & $\mathrm{p}$ & $\mathrm{r}$ \\
Organización & 17621.00 & 1.24 & .213 & - \\
Elaboración & 12505.50 & 4.05 & .000 & .21 \\
Planificación & 16152.00 & 2.56 & .010 & .13 \\
Seguimiento-Regulación & 18058.00 & .67 & .501 & - \\
Recursos ayuda & 14607.50 & 3.97 & .000 & .20 \\
Entorno estudio & 18782.50 & .19 & .848 & - \\
\hline
\end{tabular}

Los resultados indican que hay diferencias estadísticamente significativas en las estrategias de elaboración, planificación y recursos de ayuda. En los dos primeros casos el alumnado de $4 .{ }^{\circ}$ obtiene medias aritméticas superiores al alumnado de 3.. ; $\sin$ embargo, el alumnado de 3.․ declara recurrir más a ayuda en caso de dificultad que sus homólogos de $4 . .^{\circ}$ (ver tabla 3 ).

El tamaño del efecto, determinado por el coeficiente $r$, es entre débil y moderado en el caso de la elaboración y los recursos de ayuda, mientras que la diferencia en la planificación es débil.

El análisis de diferencias en el uso de estrategias de aprendizaje entre los participantes de $2 . .^{\circ}$ y de $4 .^{\circ}$ se presenta en la Tabla 6 .

Entre $2 .^{\circ}$ y $4 .{ }^{\circ}$ curso las diferencias entre puntuaciones son estadísticamente significativas en todas las estrategias analizadas ( $<<.001)$. El alumnado de $4 .^{\circ}$ obtiene puntuaciones estadísticamente superiores en las seis estrategias (ver tabla 3). El tamaño del efecto es moderado (el parámetro r está cerca del .30) en el caso de la planificación, el seguimiento-regulación, los recursos de ayuda y el entorno de estudio y entre moderado y fuerte en la organización y la elaboración. 
Tabla 6

Diferencias post-hoc entre los cursos $2 .^{\circ}$ y $4 .^{\circ}$

\begin{tabular}{ccccc}
\hline & \multicolumn{4}{l}{ Test U de Mann-Withney } \\
\hline Estrategia & $\mathrm{U}$ & $\mathrm{Z}$ & $\mathrm{p}$ & $\mathrm{r}$ \\
Organización & 10284.50 & 6.29 & .000 & .33 \\
Elaboración & 10188.00 & 7.99 & .000 & .40 \\
Planificación & 11503.00 & 5.03 & .000 & .26 \\
Seguimiento-Regulación & 11966.50 & 4.49 & .000 & .23 \\
Recursos ayuda & 11776.50 & 4.78 & .000 & .25 \\
Entorno estudio & 12110.00 & 4.43 & .000 & .23 \\
\hline
\end{tabular}

\section{Discusión y conclusiones}

El análisis de diferencias en las estrategias de aprendizaje en función del curso académico arroja datos significativos. Se evidencia un mayor uso de las estrategias de aprendizaje a medida que el curso aumenta, aunque no se produzca en todas las escalas. La variación más clara se da entre los cursos $2 .^{\circ}$ de la ESO y $3 .^{\circ}$ y entre $2 .^{\circ}$ y $4 .{ }^{\circ}$ en todas las estrategias (organización, elaboración, planificación, seguimiento-regulación, recursos de ayuda y entorno de estudio y tiempo de estudio), a excepción de la repetición.

La tendencia al alza en la utilización de estrategias a medida que avanza el curso no

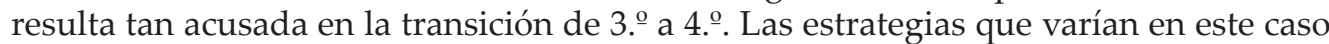
son la cognitiva de elaboración, la metacognitiva de planificación y los recursos de ayuda.

En orden a discutir las diferencias en el uso de estrategias de aprendizaje entre los cursos estudiados, cabría preguntarse si se explicitan variaciones en el propio currículo vasco para la ESO (BOPV, 2007). La realidad es que en el bloque de Matemáticas de cada curso no se hace referencia a las estrategias de aprendizaje de forma expresa, ni en los contenidos, ni en los criterios de evaluación de ninguno de ellos. Se hace mención de las estrategias como contribución de la materia a la adquisición de competencias básicas. Según dicho documento oficial, las Matemáticas ayudan al fomento de la autorregulación ("aprender a aprender") y, al mismo tiempo, se hace un llamamiento a incidir desde la materia en estrategias que faciliten el aprendizaje de manera autónoma, así como la potenciación de la perseverancia y el esfuerzo para abordar situaciones de creciente complejidad. En conclusión, según el currículo, las Matemáticas deben impulsar la autorregulación del aprendizaje.

Se constatan diferencias a favor de $4 .^{\circ}$ en la mayoría las estrategias a excepción de la repetición. A la luz de los datos, el alumnado de 4. ${ }^{\circ}$ de la ESO gestiona u organiza mejor el aprendizaje en Matemáticas (organización), relaciona los conocimientos matemáticos con otras materias de manera más competente (elaboración), conoce y regula más eficazmente los procesos cognitivos (planificación y seguimiento-regulación), recurre a solicitar ayuda más asiduamente (recursos de ayuda). También elige el lugar y el tiempo de estudio más cuidadosamente al estudiar Matemáticas (entorno y tiempo de estudio). 
Resulta especialmente reseñable la ausencia de variación en la estrategia de repetición. Se puede inferir que el alumnado que tiene un nivel de autorregulación más bajo (2.ํㅜ de la ESO) repite o memoriza en su aprendizaje matemático en la misma medida que el alumnado que hace un uso más competente de las estrategias de aprendizaje. Si se tiene en cuenta que dicha estrategia se considera superficial o de bajo nivel de procesamiento (Berger y Karabenick, 2011; Núnez et al., 1998) y que las demás estrategias tienden a subir, el dato es comprensible; esta tendencia es congruente ya que el hecho de que ciertas estrategias de orden superior se empleen más, no entra en contradicción con el estancamiento de otras que fomentan el aprendizaje pasivo produciendo un efecto negativo en el rendimiento en Matemáticas (Anthony, 1994; Kilic, Cene y Demir, 2012).

En el paso de 3. a 4. . destacan el diferencial en la escala cognitiva de elaboración, la metacognitiva de planificación y los recursos de ayuda. Reparando en la primera de ellas, se infiere que el alumnado de último curso consigue hacer un mayor empleo holístico del saber matemático, precisamente una característica intrínseca de esta materia en relación con el resto de ciencias. Dicha estrategia cognitiva es considerada de alto nivel junto a la metacognición (Berger y Karabenick, 2011) y el empleo de ambas mejora el rendimiento en Matemáticas (Kilic, Cene y Demir, 2012). Paralelamente, más allá del rendimiento, las estrategias metacognitivas mantienen una estrecha relación con el aprendizaje de las Matemáticas; el National Council of Teachers of Mathematics (NCTM) hace hincapié en que la enseñanza matemática significativa tiene como objetivo mejorar la construcción del conocimiento a través de: 1) la resolución de problemas, 2) la construcción de conexiones, 3) el desarrollo de la comunicación matemática, y 4) el uso de diversos tipos de representaciones matemáticas. En particular, este organismo realza la importancia del desarrollo metacognitivo del alumnado como medio para mejorar la resolución de problemas matemáticos y el razonamiento (NCTM, 2000).

Son escasos los estudios realizados que confronten los diversos usos de las estrategias de aprendizaje entre los cursos analizados en esta investigación. Núñez et al. (1998) afirman que el empleo de estrategias profundas de aprendizaje crece en el tránsito de los 10 a los 14 años. Zimmerman y Martínez Pons (1990) comparan los cursos de

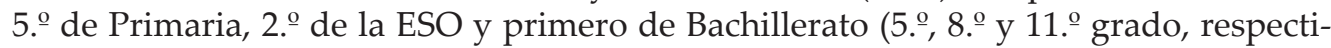
vamente) en el empleo de diversas estrategias de aprendizaje en Matemáticas, entre otras; los resultados muestran un incremento de estrategias metacognitivas de 5. o a $8 . .^{\circ}$ y un estancamiento de dichas estrategias en el paso de 8. a 11.․․ La misma progresión ocurre con la organización en el primer paso; sin embargo, de 8. . curso a 11. o curso se evidencia un descenso en esta estrategia. Por tanto, a pesar de no contar con los mismos niveles educativos, hay coincidencia en la progresión en ambas investigaciones.

Estudios más ajustados a las edades analizadas informan de un incremento con la edad del desarrollo y el control de estrategias académicas, así como del conocimiento metacognitivo en los cuatro cursos de la ESO (Inglés et al., 2013). Estos resultados concuerdan con otro investigación que da cuenta de la progresión del desarrollo cognitivo y cerebral desde finales de la niñez hasta la mitad de la adolescencia en diversos aspectos: el razonamiento deductivo, el procesamiento de la información, la experiencia, la memoria a corto y largo plazo y el conocimiento especializado (Steinberg, 2005).

Ciertas investigaciones no coinciden con los indicios encontrados en el presente estudio, aunque los niveles educativos no coincidan plenamente. Así, Rosario, Núnez, 
Valle, González-Pienda y Lourenço (2012) hallan una disminución del uso de estrategias metacognitivas en Matemáticas en alumnado entre 7. y 9. grado (1.-․․․ de la ESO), a pesar de que el peso de la diferencia es bajo. Otro estudio centrado en los dos primeros cursos de la escuela secundaria (Middle School) y que estudia estrategias cognitivas, metacognitivas y comportamentales en diversas materias, entre ellas las Matemáticas, informa de que no se dan diferencias estadísticamente significativas en la variación de cursos (Liu, 2009).

A tenor de los resultados obtenidos y de la investigación previa relativa a la comparación entre los cursos analizados, se puede afirmar que estos datos suponen una aportación al conocimiento del desarrollo del empleo de las estrategias de aprendizaje en Matemáticas en Educación Secundaria. Y, aunque no ocurra con la totalidad de las estrategias, se puede considerar una buena noticia el hecho de constatar el aumento de algunas de ellas a medida que el curso es superior, en beneficio de una mayor autorregulación del aprendizaje. Sin embargo, existe margen de mejora ya que la tendencia alcista va disminuyendo.

Sintetizando lo expuesto hasta el momento, puede decirse que las estrategias de aprendizaje en la enseñanza-aprendizaje de las Matemáticas han sido investigadas y en ciertos casos trabajadas explícitamente en el aula con el fin de mejorar el rendimiento del alumnado. De todos modos, los resultados comparativos entre cursos son escasos e incluso, en ocasiones, contradictorios. Se requiere, por tanto, profundizar en el estudio del empleo de las estrategias de aprendizaje y en su incidencia en la mejora de las competencias Matemáticas en Educación Secundaria, más allá del rendimiento.

Finalmente, conviene apuntar las limitaciones del estudio. La propia aplicación del cuestionario puede que distorsione parcialmente los resultados obtenidos; los datos concernientes al empleo de estrategias de aprendizaje han sido recopilados mediante autoinforme, con los consiguientes riesgos que eso supone para la credibilidad de los mismos. Además, el hecho de que la muestra sea de conveniencia da un matiz al sentido de los resultados. No cabe duda de que las estrategias de aprendizaje empleadas podrían depender de las características del propio alumnado, del profesorado, etc.

De igual manera, se estima importante replicar el cuestionario e implementarlo en una muestra más extensa. Esta ampliación podría ayudar a precisar mejor la consistencia de las escalas, además de contribuir a certificar la idoneidad de su validez en el sistema educativo estatal.

\section{Referencias bibliográficas}

Ahmed, W., van der Werf, G., Kuyper, H. \& Minnaert, A. (2013). Emotions, self-regulated learning, and achievement in mathematics: A growth curve analysis. Journal of Educational Psychology, 105(1), 150. http://dx.doi.org/10.1037/a0030160

Anthony, G. (1994). Learning strategies in the mathematics classroom: What can we learn from stimulated recall interviews? New Zealand Journal of Educational.

Badia, A., Álvarez, I., Carretero, R., Liesa, E. \& Becerril, L. (2012). Del aprendiz estrátegico al aprendiz competente. En A. Badia (coord.), Estrategias y competencias de aprendizaje en educación (pp.17-38). Madrid: Editorial Síntesis. 
Berger, J. \& Karabenick, S.A. (2011). Motivation and student's use of learning strategies: Evidence of unidirectional effects in mathematics classroom. Learning and Instruction, 21(3), 416-428. doi: http://dx.doi.org/10.1016/j.learninstruc.2010.06.002

BOPV (2007). Currículo de Matemáticas en la ESO. Boletín Oficial del País Vasco, suplemento al n. ${ }^{\circ} 218$.

Chiu, M. M., Wing-Yin, B. \& McBride-Chang, C. (2007). Universals and specifics in learning strategies: Explaining adolescent mathematics, science, and reading achievement across 34 countries. Learning and Individual Differences, 17, 344-365. doi: http:// dx.doi.org/10.1016/j.lindif.2007.03.007

Czuchry, M., \& Dansereau, D. F. (1998). The generation and recall of personally relevant information. Journal of Experimental Education, 66(4), 293-315. doi: http://dx.doi. org/10.1080/00220979809601403

Dinsmore, D. L., Alexander, P. A. \& Loughlin, S. M. (2008). Focusing the conceptual lens on metacognition, self-regulation, and self-regulated learning. Educational Psychology Review, 20, 391-409. http://dx.doi.org/10.1007/s10648-008-9083-6

European Commission (2006). Key competences for lifelong learning: A European Reference Framework. Brussels: European Commission.

Field, A. (2009). Non-parametric tests. En Discovering statistics using SPSS (pp. 539-583, 3 ${ }^{a}$ edición). London: Sage. doi: http://dx.doi.org/10.1002/bjs.7040

García-García, M., Biencinto-López, C., Carpintero-Molina, E.; Núñez-del-Río, M.C. \& Arteaga- Martínez, B. (2013). Rendimiento en Matemáticas y actitud hacia la materia en centros inclusivos: estudio en la Comunidad de Madrid. Revista de Investigación Educativa, 31(1), 117-132. doi: http://dx.doi.org/10.6018/rie.31.1.143221

Gargallo, B. (2012). Un aprendiz estratégico para una nueva sociedad. Tesi 13(2), 246-272.

Gil-Jaurena, I. (2012). Observación de procesos didácticos y organizativos de aula en Educación Primaria desde un enfoque intercultural. Revista de Educación, 358, 85-110.

Inglés, C. J., Martínez-González, A. E. \& García-Fernández. J. M. (2013). Conducta prosocial y estrategias de aprendizaje. European journal of educational psychology, 6(1), 33-53.

Kilic, S., Cene, E. \& Demir, I. (2012). Comparison of Learning Strategies for Mathematics Achievement in Turkey with Eight Countries. Educational Sciences: Theory $\mathcal{E}$ Practice, 12(4), 2594-2598.

Liu, O. L. (2009). Evaluation of a learning strategies scale for middle school students. Journal of Psychoeducational Assessment, 27(4), 312-322. doi: http://dx.doi. org/10.1177/0734282908327935

Marugán, M., Carbonero, M.A., León, B. \& Galán, M. (2013). Análisis del uso de estrategias de recuperación de la información por alumnos con alta capacidad intelectual (9-14 años) en función del género, edad, nivel educativo y creatividad. Revista de Investigación Educativa, 31 (1), 185-198. doi: http://dx.doi.org/10.6018/rie.31.1.147361

Monereo, C. \& Badia, A. (2013). Aprendizaje estratégico y tecnologías de la información y la comunicación: una revisión crítica. Tesi, 14(2), 15-41.

Murayama, K., Pekrun, R., Lichtenfeld, S. \& Vom Hofe, R. (2013). Predicting LongTerm Growth in Students' Mathematics Achievement: The Unique Contributions of Motivation and Cognitive Strategies. Child development, 84(4), 1475-1490. doi: http:// dx.doi.org/10.1111/cdev.12036 
National Council of Teachers of Mathematics (NCTM) (2000). Principles and standards for school mathematics. Reston, VA. doi: http://dx.doi.org/10.1177/019263650108562305

Nisbet, J. \& Shucksmith, J. (1986). Estrategias de aprendizaje. Madrid: Santillana.

Núñez, J.C., González-Pienda, J.A., García, M.S., González-Pumariega, S. \& García, S.I. (1998). Estrategias de aprendizaje en estudiantes de 10 a 14 años y su relación con los procesos de atribución causal, el autoconcepto y las metas de estudio. Estudios de Psicología, 59, 65-85. doi: http://dx.doi.org/10.1174/02109399860400739

Perels, F., Dignath, C. \& Schmitz, B. (2009). Is it possible to improve mathematical achievement by means of self-regulation strategies? Evaluation of an intervention in regular math classes. European Journal of Psychology of Education, 24(1), 17-31. doi: http://dx.doi.org/10.1007/bf03173472

Perels, F., Gürtler, T. \& Schmitz, B. (2005). Training of self-regulatory and problem-solving competence. Learning and Instruction, 15, 123-139. doi: http://dx.doi.org/10.1016/j. learninstruc.2005.04.010

Pintrich, P. R. (1995). Understanding self-regulated learning. San Francisco: Jossey-Bass. doi: http://dx.doi.org/10.1002/tl.37219956304

Pintrich, P. R., Smith, D. A., García, T. \& McKeachie, W. J. (1991). A Manual for the Use of the Motivational Strategies for Learning Questionnaire (MSLQ). Ann-Arbor, MINCRIPTAL: The University of Michigan. doi: http://dx.doi.org/10.1177/0013164493053003024

Rosario, P., Núñez, J. C., Valle, A., González-Pienda, J. \& Lourenço, A. (2012). Grade level, study time, and grade retention and their effects on motivation, self-regulated learning strategies, and mathematics achievement: a structural equation model. European journal of psychology of education, 28(4), 1311-1331. doi: http://dx.doi.org/10.1007/ s10212-012-0167-9

Steinberg, L. (2005). Cognitive and affective development in adolescence. Trends in Cognitive Sciences, 9, 69-74. doi: http://dx.doi.org/10.1016/j.tics.2004.12.005

Thoutenhoofd, E. D. \& Pirrie, A. (2013). From self-regulation to learning to learn: observations on the construction of self and learning. British Educational Research Journal. doi: http://dx.doi.org/10.1002/berj.3128

Thiessen, V. \& Blasius, J. (2008). Mathematics achievement and mathematics learning strategies: Cognitive competencies and construct differentiation. International Journal of Educational Research, 47, 362-371. doi: http://dx.doi.org/10.1016/j.ijer.2008.12.002

Wernke, S., Wagener, U., Anschuetz, A. \& Moschner, B. (2011). Assessing cognitive and metacognitive learning strategies in school children: Construct validity and arising questions. International Journal of Research and Review, 6(2), 19-38.

Zamani, A. \& Mir, M. (2011). Surveying the Effect of Metacognitive Education on the on the Mathematics Achievement of $1^{\text {st }}$ Grade High Junior School Female Students in Educational District 5, Tehran City, 2009-10 Educational Year. Procedia-Social and Behavioral Sciences, 29, 1531-1540.doi: http://dx.doi.org/10.1016/j. sbspro.2011.11.394

Zimmerman, B. J. (1994). Dimensions of Academic Self-Regulation: A Conceptual Framework for Education. Hillsdale, New Jersey: Lawrence Erlbaum.

Zimmerman, B. J. \& Martínez-Pons, M. (1990). Student differences in self-regulated learning: Relating grade, sex and giftedness to self-efficacy and strategy use. Journal of Educational Psychology, 82, 51-59. doi: http://dx.doi.org/10.1037/0022-0663.82.1.51 


\section{Anexo}

Cuestionario de estrategias en el aprendizaje de las Matemáticas

\begin{tabular}{|c|c|}
\hline R1 & $\begin{array}{l}\text { Cuando estudio Matemáticas repito lo que necesito aprender una y otra vez para memo- } \\
\text { rizarlo }\end{array}$ \\
\hline 2 & $\begin{array}{l}\text { Para estudiar Matemáticas repito varias veces las fórmulas o definiciones con el fin de } \\
\text { memorizarlas }\end{array}$ \\
\hline 3 & Cuando estudio Matemáticas suelo repetir varias veces los problemas para memorizarlos \\
\hline 1 & $\begin{array}{l}\text { Estudio Matemáticas haciendo diagramas, cuadros o tablas para organizar lo que he } \\
\text { aprendido }\end{array}$ \\
\hline 2 & $\begin{array}{l}\text { Cuando estudio Matemáticas hago una lista de las fórmulas o definiciones para organizar } \\
\text { lo que necesito aprender }\end{array}$ \\
\hline 1 & Cuando estudio Matemáticas intento relacionar lo nuevo con lo que ya sé \\
\hline 12 & $\begin{array}{l}\text { Relaciono la manera de resolver problemas matemáticos con la manera de resolver otros } \\
\text { problemas }\end{array}$ \\
\hline 1 & Antes de estudiar un nuevo tema planeo cómo voy a hacerlo \\
\hline 12 & Antes de empezar a estudiar Matemáticas planeo qué y cómo voy a hacerlo \\
\hline 13 & $\begin{array}{l}\text { Antes de empezar a estudiar Matemáticas pienso cuánto tiempo voy a necesitar para es- } \\
\text { tudiar el tema }\end{array}$ \\
\hline 14 & $\begin{array}{l}\text { Cuando estudio un nuevo tema de Matemáticas pienso cuál puede ser la mejor manera } \\
\text { de hacerlo }\end{array}$ \\
\hline 5 & Antes de estudiar Matemáticas me fijo objetivos que me ayudan a aprender \\
\hline 1 & Cuando estudio Matemáticas me hago preguntas para estar seguro de lo que \\
\hline 2 & Cuando estudio Matemáticas llevo un control de lo que he aprendido \\
\hline 3 & Cuando estudio Matemáticas me pregunto si conozco los materiales \\
\hline 24 & Compruebo si he comprendido lo que estoy aprendiendo \\
\hline e1 & Si no entiendo algo que estoy estudiando en Matemáticas intento solucionarlo \\
\hline $\mathrm{e} 2$ & Si las Matemáticas que estoy estudiando son difíciles de aprender, me lo tomo con calma \\
\hline e3 & Si tengo dificultades para resolver un problema, intento otras formas para resolverlo \\
\hline n1 & Estudio Matemáticas en un entorno en el que puedo concentrarme \\
\hline En2 & Me hago un horario para preparar los exámenes de Matemáticas \\
\hline En3 & Estudio Matemáticas a una hora en la que puedo concentrarme \\
\hline Ay1 & Si no entiendo algo en Matemáticas pido ayuda a mi profesor o profesora \\
\hline Ay2 & Si no entiendo algo en Matemáticas pido ayuda a otros estudiantes \\
\hline$y 3$ & Si no entiendo algo en Matemáticas recurro a otros materiales \\
\hline
\end{tabular}

R: Repetición; O: Organización; El: Elaboración; Pl: Planificación; Se: Seguimiento; Re: Regulación; En: Entorno-tiempo de estudio; Ay: Recursos de ayuda

Fecha de recepción: 13 de marzo de 2015.

Fecha de revisión: 13 de marzo de 2015.

Fecha de aceptación: 29 de marzo de 2016. 